\title{
Effective Modeling of Leakage Inductance for use in Circuit Simulation
}

\begin{abstract}
Theoretical methods for the calculation of winding losses with respect to frequency have been well documented, but the variation in leakage inductance of magnetic components due to frequency has been less well addressed. In this paper Dowell's wellknown theoretical approach is tested using measurements and finite element analyses. The results presented show deficiencies in the accuracy of models generated using Dowell's approach and highlight the trade-offs between model complexity, simulation time and accuracy when the finite element analysis method is applied. An alternative behavioural model is presented which models the frequency variation in leakage inductance accurately, is easy to characterize from simple measurements or calculations and is robust.
\end{abstract}

\section{INTRODUCTION}

Extensive theoretical and empirical research has taken place to establish the variation of winding losses with frequency. Dowell [1] provides a theoretical framework for the calculation of losses in transformer windings, and this has been developed by a large number of authors since including Cheng and Evans [2],[3]. Roberts, Mathys and Schauwers [4] have studied the accuracy of the theory and have noted discrepancies between the theoretical and empirical values of losses obtained while Niemela et al [5] have extended the theory for general multiple winding topologies. There has been much less effort applied to the equivalent theoretical calculation of leakage inductance, especially at higher frequencies where eddy current and proximity effects become significant. The modeling of the leakage inductance of magnetic components and its variation with frequency has been addressed theoretically by Dowell [1] and by Dauhajre [6], Dauhajre \& Middlebrook [7] and Hsu, Middlebrook and Cuk [8]. Of these, however, only Dowell provides a theoretical approach for the estimation of the combined eddy current and proximity effects and the resulting leakage inductance variation with frequency. More recent work by Hurley and Wilcox [9] has provided an alternative approach, but is specifically for toroidal transformers. The problem with all of these methods is the inaccuracy of the predicted values for leakage inductance as the frequency increases. In general, the focus of the effort to characterize the high frequency behaviour of windings, in particular leakage inductance, has been concentrated on the application of finite element analysis, with Silvester \& Konrad [10], Tabaga, Pierratt \& Blache [11], Skutt, Lee, Ridley \& Nicol [12], Dai \& Lee [13]-[14] and Cherpentier, Lefevre \& Lajoie-Mazenc [15] demonstrating the approach. The methodology is now well understood and is often provided as an application example in commercial finite element analysis software packages.

The development of the finite element analysis software as the integral part of a procedure to calculate the high frequency behaviour of magnetic components has been developed extensively by Asensi et al [16]-[18] and Prieto [19]-[21]. This procedure has the function of providing models for practical electric circuit simulation of power applications, and this has also been addressed by Okyere \& Heinemann [22] and Evans \& Heffernan [23]. Unfortunately the procedure requires exhaustive finite element analyses of the transformer models, which become more finely meshed as the frequency increases thereby either increasing the simulation time required, or reducing the accuracy of the result. The resulting model obtained may also be cumbersome and exhibit convergence difficulties. Lopera et al [26] and Pernia et al [27],[28] have developed a onedimensional approximation of the behaviour of the magnetic component windings including frequency variations. This has been developed into a software package UO- $\mathrm{M}^{2} \mathrm{~T}$ by Prieto et al [29] that produces a netlist model of the magnetic component for use in circuit simulation. There are two issues to consider with this type of approach. The first is the size and complexity of the resulting model. If the model consists of a large number of elements, then this may impact on the convergence and time of the circuit simulation. The second issue is the de-coupling of the model elements from the original physical geometry. If a model can be constructed which retains this link, then sensitivity of key parameters such as interlayer gap or winding size can be usefully investigated without the requirement for a remodeling of the device. This technique is discussed specifically in this paper. In this paper a model structure is proposed which models the leakage inductance variation with frequency, and can be characterized with a relatively small number of measurements or finite element analyses.

\section{EVALUATION OF DOWELL’s EQUATIONS FOR THE PREDICTION OF LEAKAGE INDUCTANCE}

If the theoretical equations of Dowell [1] and Dauhajre et al [6]-[7] are evaluated, it can be shown that the low frequency leakage inductance is effectively obtained from the same equation, the apparent differences are purely a matter of nomenclature. If a two-winding transformer is taken as an example, Dauhajre provides the expression for the leakage inductance based on this geometry. 


$$
l=2 \pi \mu_{0} N_{1}^{2} \frac{1}{b}\left[\begin{array}{l}
h_{1}\left(\frac{r_{0}}{3}+\frac{h_{1}}{4}\right) \\
+\delta\left(r_{0}+h_{1}+\frac{\delta}{2}\right) \\
+h_{2}\left(\frac{r_{0}+h_{1}+\delta}{3}+\frac{h_{2}}{12}\right)
\end{array}\right]
$$

This approach is fundamentally the same as Dowell's in that the energies stored in each winding space and the interwinding spaces are calculated independently and then combined to provide the complete leakage inductance value, referred to a single winding (e.g. the primary). Dauhajre stops at this point, but Dowell makes the point that by dividing the energy regions into winding and spaces, the winding regions are frequency dependent, due to eddy currents and proximity effects, but the spaces are not. For a two winding transformer, the overall expression for the leakage inductance is given from Dowell's equations by (2), where $L_{w 1}$ is the leakage inductance in winding $1, L_{w 2}$ is the leakage inductance in winding $2, F_{L 1}$ and $F_{L 2}$ are the frequency variations in the leakage indutances of winding 1 and 2 respectively, $L_{g}$ is the leakage inductance due to the interwinding gap and $L_{U}$ is the leakage inductance due to the interlayer gaps.

$$
L_{\text {leakage }}=F_{L 1} L_{w 1}+F_{L 2} L_{w 2}+L_{g}+L_{U}
$$

Where the frequency variation of each winding leakage inductance is represented by a lumped equation for all of the eddy current and proximity effects as shown by (3)-(6).

$$
F L=\left\{\frac{3 M^{\prime \prime}+\left(m^{2}-1\right) D^{\prime \prime}}{m^{2}\left|\alpha^{2} h^{2}\right|}\right\}
$$

Where $M^{\prime \prime}$ is the imaginary part of the function $M$ given in (4), and $D^{\prime \prime}$ is the imaginary part of the function $D$ given in (5). $\alpha$ is the expression for effective skin depth given in (6), $m$ is the number of winding layers and $h$ is the layer thickness.

$$
\begin{gathered}
\boldsymbol{M}=\alpha \boldsymbol{h} \operatorname{coth} \alpha \boldsymbol{h} \\
\boldsymbol{D}=2 \alpha \boldsymbol{h} \tanh \frac{\alpha \boldsymbol{h}}{2} \\
\alpha=\sqrt{\frac{\boldsymbol{j} \boldsymbol{w} \mu_{0} \eta}{2}}
\end{gathered}
$$

To assess the accuracy of these equations, a 2 winding transformer was constructed using a Phillips RM12-3F3 core, and wound using 24 SWG ( $0.559 \mathrm{~mm}$ diameter) enameled copper wire with both the primary and secondary having 2 layers of 21 turns each. The inter-winding gap was measured to be $1.2 \mathrm{~mm}$. The leakage inductance was obtained by measuring the impedance across the primary, while short-circuiting the secondary winding. An HP4192A impedance analyzer was used, varying the frequency from $100 \mathrm{~Hz}$ to $1 \mathrm{MHz}$. The short circuit wires on the secondary winding were kept to a minimum length to reduce the introduction of extraneous leakage due to the measurement set up. The correlation of the measured and calculated leakage inductance values at $1000 \mathrm{~Hz}$ seems to indicate the shorting wires have little impact on the measured leakage inductance. The low frequency leakage inductance was calculated using Dauhajre's equation (1) and verified using Dowell's approach with the value estimated at $12.52 \mathrm{uH}$. A Mathcad model was then created for equation (3) and the packing factor $(\eta)$ varied using 1.0 (perfect winding area coverage), 0.866 (perfect round conductor packing) and 0.5 (practical estimate). The resulting variation of measured and theoretical values is given in figure 1.

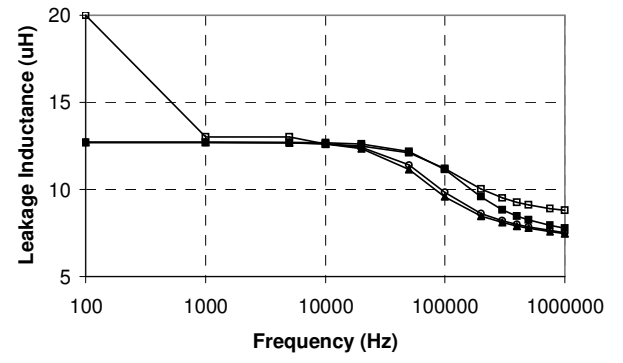

- Measured - Dowell $\mathrm{n}=0.5 \multimap$ Dowell $\mathrm{n}=1.0 \multimap-$ Dowell $\mathrm{n}=0.866$

Figure 1: Theoretical and empirical leakage inductance variation with frequency

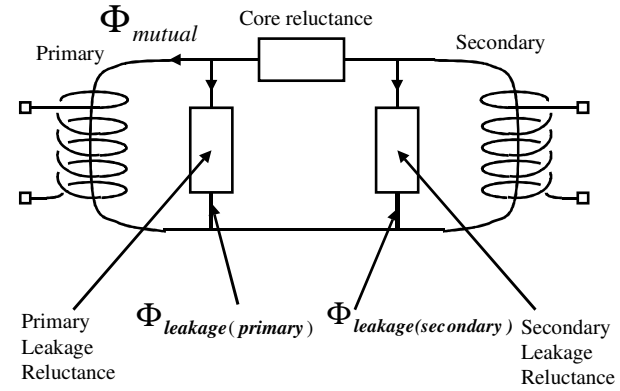

Figure 2: Electro-Magnetic Transformer Model 
There are two interesting points to note from figure 2. The first is that as the frequency drops to around $100 \mathrm{~Hz}$, the apparent leakage inductance seems to increase. The explanation for this, is that as the frequency drops, the impedance of the magnetizing inductance falls and becomes comparable to the winding resistance. This has the consequence that the measured inductance can no longer be assumed to be an accurate measure of the leakage inductance alone. This causes the measured leakage inductance to apparently increase. The second point to notice is that as the frequency increases, the predicted leakage inductance decreases more quickly than the measured value. Niemela et al [5] and Robert et al [4] have also observed this difference. It is also noticeable that as the frequency tends to $1 \mathrm{MHz}$, the leakage inductance flattens off to a high frequency value. In summary, therefore, it is clear that Dowell's theory is not always accurate at higher frequencies at predicting the roll off of the leakage inductance, or its final high frequency value. This is probably due to the analysis by Dowell being effectively carried out in one dimension and not considering two dimensional effects.

\section{PROPOSED NEW MODEL FOR LEAKAGE INDUCTANCE}

Given the deficiencies in both the theoretical and finite element analysis approaches demonstrated previously, there is the requirement for a simple model of the frequency variation of leakage inductance, which can reasonably accurately (to within $10 \%$ ) model the behaviour of the leakage inductance with frequency which can be characterized with either a few measurements or finite element analyses. A further requirement of the model was to provide a simple approach that would allow direct understanding of the effect specific model parameters would have on the circuit performance with minimal simulation overhead. A new model structure is therefore proposed which reasonably models the low and high frequency behaviour of leakage inductance, and the transition between these two states. If the model of a transformer is considered in the magnetic domain, as shown in figure 2, the component consists of two windings, and a magnetic circuit containing the core and leakage reluctances.

It is proposed to split each leakage reluctance into two parts, a d.c. or low frequency Reluctance $\left(v_{d c}\right)$ and a high frequency Reluctance $\left(v_{h f}\right)$. At low frequencies the total reluctance is the parallel combination of $v_{d c}$ and $v_{h f}$ while at high frequencies the effect of the d.c. reluctance is reduced by eddy currents represented by the inductance $L_{\text {Loss }}$, so the total reluctance tends towards the high frequency value $v_{h f}$. The inductance $L_{\text {Loss }}$ in the magnetic domain is equivalent to a closed turn linked to the flux as described by Cherry [24] and Laithwaite [25]. The time constant of the $v_{d c}$ reluctance and the loss inductor is effectively obtained from the conductor diameter. The resulting model of leakage reluctance is given in figure 3 .

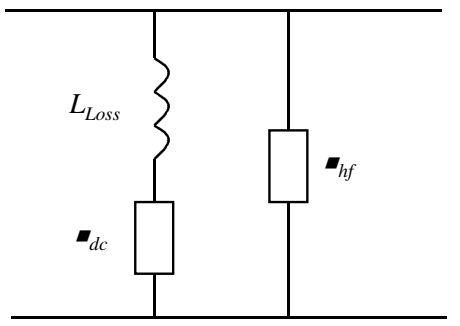

Figure 3: Modified model of leakage reluctance

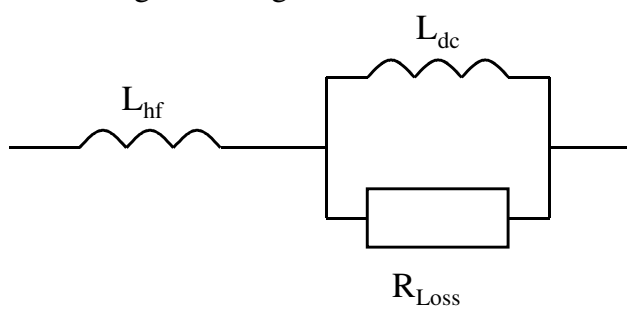

Figure 4: Proposed Leakage Inductance Model

This model can also be transformed into an equivalent in the electrical domain [24] using the configuration shown in figure 4. The $L_{\text {Loss }} \& v_{d c}$ (or $R_{\text {Loss }} \& L_{d c}$ using the electrical model) time constant can be estimated by using the frequency at which the skin depth is 0.5 the radius of the conductors used. This gives a reasonable estimate of the cut off frequency from which the loss component can be calculated. The low and high frequency leakage reluctances (or the inductances in electrical domain) can be measured or calculated using a high frequency and low frequency test thus reducing the number of tests (or finite element analyses) drastically. An alternative theoretical approach is to use Dauhajre's or Dowell's equations which separate the frequency and non-frequency dependent sections of the leakage inductance. Using this approach, the proportion of the leakage inductance due to the high frequency effect can be estimated.

The model of the transformer was constructed in SPICE as shown in figure 13, using the estimate of time constant based on the skin depth at half the conductor radius and the comparison with the measured leakage inductance given in figure 14. The ideal form of transformer model was used, with a measured magnetizing inductance of $10 \mathrm{mH}$ and perfect coupling $(k=1)$. The low frequency leakage inductance was measured at $13 \mathrm{uH}$, and the eventual effective high frequency leakage inductance was measured at $8.8 \mathrm{uH}$. Thus $L_{h f}$ was set to $8.8 \mathrm{uH}$ and $L_{d c}$ was set to the difference between the low and high frequency leakage inductance measurements, giving a value of $4.2 \mathrm{uH}$. In this model, the parasitic components were split evenly between the two windings, although the same value of leakage would be obtained by referring the values to one winding. The winding resistance 
$R_{w}$ was measured using a resistance bridge. The estimation of the $R_{\text {loss }}$ resistance, made with the skin depth at half the conductor radius, which can be estimated for 24 s.w.g. wire to be a depth of $0.13975 \mathrm{~mm}$, occurring at $223.6 \mathrm{kHz}$. Using this frequency, and the previously measured leakage inductance of $13 \mathrm{uH}$, the $R_{\text {loss }}$ value is estimated to be $2.9 \Omega$ using a simple RL time constant calculation. While this gives a reasonable estimate of the frequency variation, it is also the case that with such a simple model the accuracy of the final result may not be always optimal. It is an advantage of the simple model however, that improvements can be made in the matching with measured results by small variations in the value of $R_{\text {loss }}$. By including the magnetizing inductance and winding resistances in the model, the correct low frequency behaviour was also observed. The test circuit shown in figure 5 used a current source on the primary side, and effectively shorted out the secondary winding using a $1 \mathrm{~m} \Omega$ resistor. The imaginary part of the input voltage vin was then divided by $2 \pi f$ to give the effective leakage inductance shown in figure 6 . The magnetizing inductance was obtained by measurement, with a value of $10 \mathrm{mH}$.
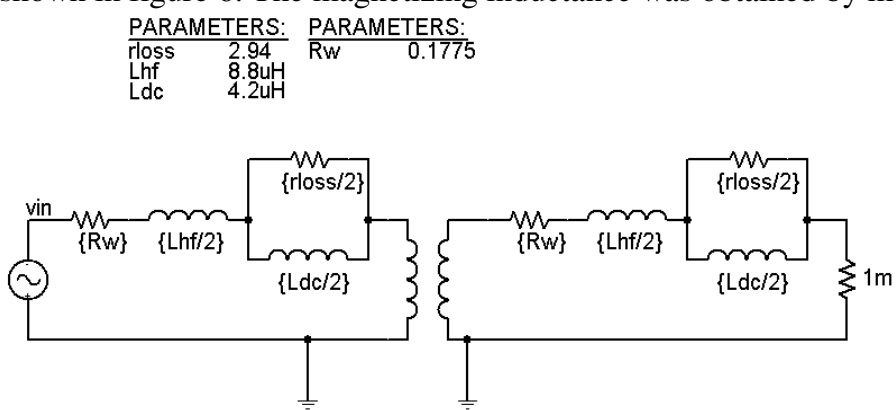

Figure 5: Test Circuit for transformer, including the modified leakage inductance model, implemented in Spice

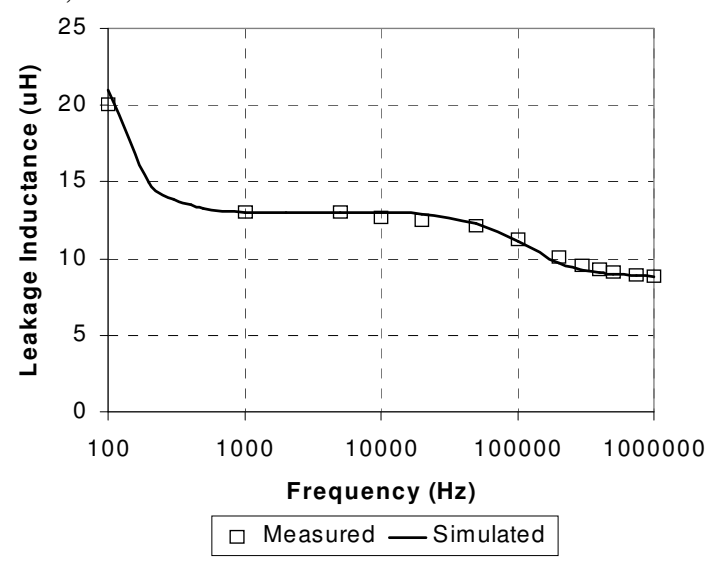

Figure 6: Measured and Simulated leakage Inductance

\section{CONCLUSIONS}

Testing of Dowell's theory of the variation of leakage inductance with frequency has demonstrated limitations in the accuracy of the method at high frequencies. Study of the finite element analysis approaches to the calculation of leakage inductance has also highlighted difficulties in the practical application of this method to the effective calculation of leakage inductance variation with frequency in a reasonable time. A new model structure has been presented which accurately models the variation of leakage inductance with frequency using a simple approach. The resulting model is simple to characterize, stable and fast to simulate.

\section{REFERENCES}

[1] P. Dowell, "Effects of eddy currents in transformer windings", Proceedings of the IEE, Vol. 113, No 8, 1966, p1387

[2] K.W.E. Cheng \& P.D. Evans, Calculation of winding losses in high frequency toroidal inductors using single strand conductors, IEE Proceedings - Electric Power Applications, Vol. 141, No 2, Mar 1994, pp52-62

[3] K.W.E. Cheng \& P.D. Evans, Calculation of winding losses in high frequency toroidal inductors using multi-strand conductors, IEE Proceedings - Electric Power Applications, Vol. 142, No 5, Sep 1995, pp313-322

[4] F. Robert, P. Mathys \& J.P. Schauwers, "Ohmic Losses Calculation in SMPS Transformers: numerical study of Dowell's approach accuracy”, IEEE Transactions on Magnetics, Vol 34, No. 4, July 1998, pp1255-7

[5] Niemela V.A., Skutt G.R., Urling A.M., Chang Y.N., Wilson T.G., Owen H.A., Wong R.C., "Calculating the short-circuit impedance of a multiwinding transformer from its geometry”, IEEE Power Electronics Specialists Conference (PESC), Vol 2, 1989, p 607-617

[6] A. Dauhajre, "Modeling and Estimation of Leakage Phenomena in Magnetic Circuits", PhD Thesis, California Institute of Technology, Pasadena, California, 1986.

[7] A. Dauhajre \& R.D. Middlebrook, Modeling and estimation of leakage phenomenon in magnetic circuits, IEEE Power Electronics Specialists Conference (PESC), 1986, pp 213-226

[8] S.P. Hsu , R.D. Middlebrook \& S. Cuk, "Transformer modeling and design for leakage control", Advances in SwitchedMode Power Conversion, 2nd Edition, TESLAco, 1983, Chapter 13, pp205-218.

[9] Hurley W.G., Wilcox D.J., “Calculation of Leakage Inductance in Transformer Windings”, IEEE Transactions on Power Electronics, Vol. 9, No. 1, January 1994, pp121-126 
[10] P.P. Silvester \& A. Konrad, Analysis of transformer leakage phenomena by high order finite elements , IEEE PES, 1973, p1843

[11] S. Tabaga, L. Pierrat \& F. Blache, "Parameter Computation of a planar transformer by 3D Finite Element Method", EPE Conference Proceedings, Seville, 1995, pp 1.273-276

[12] G. Skutt, F.C. Lee, R. Ridley \& D. Nicol, "Leakage Inductance and Termination Effects in a High Power Planar Magnetic Structure", IEEE Applied Power Electronics Conference and Exposition - APEC, Vol. 1, 1994, pp 295-301

[13] Dai N., Lee F.C., "Characterization and Analysis of Parasitic Parameters and their effects in Power Electronics Circuit", IEEE Annual Power Electronics Specialists Conference (PESC), Vol. 2, (1996), pp 1370-1375

[14] Dai N., Lee F.C., "High-Frequency Eddy-Current Effects in Low-Profile Transformer Windings”, IEEE Annual Power Electronics Specialists Conference, v 1, (1997), pp 641-647

[15] Charpentier J.F., Lefevre Y., Lajoie-Mazenc M., "A 2D Finite Element Formulation for the study of the high frequency behaviour of wound components”, IEEE Transactions on Magnetics, Vol. 32, No. 3, May 1996, pp 1098-1101

[16] R. Asensi, J.A. Cobos, O. Garcia, R. Prieto \& J Uceda, New Modeling Strategy for high frequency transformer windings, IECON Proceedings, Vol. 1, (1995), pp 246-251

[17] R. Asensi, J.A. Cobos, O. Garcia, R. Prieto \& J Uceda, A full procedure to model high frequency transformer windings, IEEE Power Electronics Specialist Conference Proceedings, Vol. 2, 1994, pp 856-863

[18] R. Asensi, J.A. Cobos, O. Garcia, R. Prieto \& J Uceda, A CAD tool for magnetic components modeling, IEEE Applied Power Electronics Conference and Exposition - APEC, Vol. 1, 1996, pp 427-433

[19] R. Prieto, J.A. Cobos, O. Garcia, P. Alou \& J. Uceda, "Taking into account all the parasitic effects in the design of magnetic components", APEC '98, Vol. 1, 1998, pp400-406

[20]R. Prieto, J.A. Cobos, O. Garcia \& J. Uceda, "Interleaving techniques in magnetic components", APEC '97, Vol. 2, pp931-936

[21]R. Prieto, J.A. Cobos, O. Garcia \& J. Uceda, "Influence of the winding strategy on the parasitics of magnetic components", Proceedings EPE '97, Vol. 2, pp38-43

[22] Okyere P.F. \& Heinemann L., "An Advanced Spice-Compatible Model for High Frequency Multiwinding Transformers", IEEE Annual Power Electronics Specialists Conference (PESC), Vol. 1, 1999, pp 592-597

[23] Evans P.D., Heffernan W.J.B., "Transformer for multimegahertz power applications", IEE Proceedings on Electric Power Applications, Vol. 142, No. 6, November 1995, pp379-389

[24]E.C. Cherry, "The duality between inter-linked electric and magnetic circuits", Proceedings of the Physics Society, Volume 62, 1949 pp101-111

[25]E. R. Laithwaite, "Magnetic Equivalent Circuits for electrical machines", PROC. IEE, Vol. 114, November 1967, pp18051809

[26] J. M. Lopera, A. M. Pernía, J. Díaz, J. M. Alonso and F. Nuño, “A complete transformer Electric Model, including Frequency and Geometric Effects", IEEE Power Electronics Specialists Conference, Toledo (Spain), June-July 1992, pp1247-1252

[27] A. M. Pernía, F. Nuño and J. M. Lopera, "Magnetic Elements Simulation in Power Converters”, Congreso Internacional de Electrónica de Potencia, Puebla (Mexico), Aug. 1994, pp74-79

[28] A. M. Pernía, F. Nuño and J. M. Lopera, “1D/2D Transformer Electric Model for Simulation in Power Converters”, IEEE Power Electronics Specialists Conference, Atlanta (USA), June 1995, pp. 1043-1049

[29] M.J. Prieto, J.M Lopera, A.M. Pernía, F.F. Linera and F. Nuño, "High-Frequency magnetic elements design tool", IEEE Workshop on Computers in Power Electronics (COMPEL '98), Villa Erba (Italy), 1998, pp. 127-131 